\title{
ORIGINAL
}

\section{BROTE DE PSITACOSIS EN GRANADA}

\author{
Ma Teresa León Espinosa de los Monteros (1), Juan Antonio Laguna Sorina (2), Ma Trinidad Rue- \\ da Domingo (3), Begoña López Hernández (4), Mª José Bermejo Pérez (1) y José Carlos Sabonet (4). \\ (1) Servicio de Medicina Preventiva. Hospital Universitario Virgen de las Nieves de Granada \\ (2) Sección de Epidemiología de la Delegación Provincial de Salud de Granada. \\ (3) Epidemiología y Programas. Distrito Sanitario Aljarafe. Sevilla \\ (4) Distrito Sanitario de Granada.
}

\section{RESUMEN}

Fundamento: La psitacosis se puede transmitir de pájaros infectados a seres humanos, siendo causa de neumonía atípica. El objetivo de este trabajo es determinar origen de un brote de psitacosis, las características de los sujetos expuestos, y describir las medidas para controlarlo.

Métodos: Estudio descriptivo. Comunicación al Sistema de Vigilancia Epidemiológica y Delegación de Agricultura, realizándose las actuaciones pertinentes (inspección, toma de muestras, desinfección e inmovilización cautelar de aves), clausura del estableci miento; definición de caso y encuesta epidemiológica. Análisis de frecuencias.

Resultados: 17 personas tuvieron contacto con animales sospechosos (53\% varones/47\% mujeres), de ellos 9 (edad media: 30 ) presentaron síntomas ( $100 \%$ fiebre, cuadro pseudogripal $44,4 \%$ ). Se realizó serología para C. psitacci a 11 de los 17 sujetos expuestos $(68,75 \%)$, resultando positiva en dos de ellos $(18,18 \%)$. En el estudio de tejido pulmonar del fallecido se comprobó la presencia de DNA de C. psitacci. La confirmación diagnóstica entre personas sintomáticas fue del 33\% (3/9). La Delegación de Agricultura impuso cuarentena a 22 núcleos zoológicos. De las 70 muestras tomadas (60: cloacas de animales y 10 aves), presentaron antígeno positivo 7 $(10 \%)$, siendo la detección de ácidos nucleicos por PCR positiva en una de ellas.

Conclusiones: La exposición a aves enfermas en los establecimientos implicados originó el brote, siendo el foco común de contagio de carácter laboral. La adecuada coordinación/colaboración entre las Delegaciones de Salud y Agricultura permitió evitar la aparición de casos nuevos.

Palabras clave: Psitacosis. Brote epidémico. Ornitosis. Zoonosis. España

Correspondencia:

$\mathrm{M}^{\mathrm{a}}$ Teresa León Espinosa de los Monteros

Camino de Ronda $166,2^{\circ} \mathrm{A}$.

18003 Granada

Correo electrónico: mayte@moebius.es
ABSTRACT

\section{Psittacosis Outbreak in Granada, Spain}

Background: Psittacosis can be transmitted to humans from infected birds, being the cause of atypical pneumonia. This work is aimed at determining the source of a psittacosis outbreak, the characteristics of the subjects exposed and describing the measures to control the outbreak.

Methods: Descriptive study. Notification to the Epidemiological Monitoring System and Autonomic Agriculture authorities, taking the pertinent measures (inspection, sampling, disinfecting and precautionary quarantine of birds), closing the establishment. Case definition and epidemiological survey. Frequency analysis.

Results: Seventeen (17) individuals (one of whom died) came into contact with suspicious animals (53\% males/47\% females), nine of whom (mean age: 30 ) showed symptoms (100\% fever, flulike symptoms $44.4 \%$ ). A serology was performed for C. psitacci on 11 of the 17 subjects exposed $(68.75 \%)$, two of whom $(18.18 \%)$ tested positive. The presence of C. psittaci DNA was detected in the pulmonary tissue study conducted on the deceased individual. A total of 33\% (3/9) of those showing symptoms were confirmed by diagnosis. The Autonomic Agriculture authorities placed 22 animal compounds under quarantine. A total of seven (10\%) of the 70 samples taken (60: animal cloacas and 10 birds) revealed a positive antigen, the PCR nucleic acid test being positive in one thereof.

Conclusions: The exposure to diseased animals at the establishments involved caused the outbreak, the common source of contagion being of an occupational nature. The appropriate coordination/collaboration between the local Health and Agriculture authorities made it possible to prevent any further cases from arising.

Key words: Psittacosis. Outbreaks. Ornithosis. Zoonose. España. 


\section{INTRODUCCIÓN}

La psitacosis es una enfermedad zoonótica, causada por la bacteria Chlamydiophila psitacci, parásito intracelular obligado. En la actualidad se reconocen cuatro especies pertenecientes a este género $(C$. pecorum, $C$. psitacci, C. trachomatis, C. pneumoniae $)^{1}$.

La psitacosis se puede transmitir de pájaros infectados a los seres humanos, siendo una causa importante de infección sistémica que a menudo provoca neumonía. La distribución de la enfermedad es mundial, siendo el reservorio principal loros, periquitos, cotorras, papagayos y con menor frecuencia aves de corral, palomas, canarios y aves marinas $^{2-3}$.

La incidencia estimada de la neumonía por $C$. psitacci es escasa, situándose en distintas regiones españolas entre el $1 \%$ y el $5 \%$ de las neumonías de la comunidad que ingresan en el hospital. En otros países de la Unión Europea el porcentaje puede ascender al 7\%-11\%. La mortalidad de los pacientes tratados es inferior al $1 \%{ }^{4}$.

En Andalucía, como en el resto de España ${ }^{5}$, la psitacosis no es una enfermedad de declaración obligatoria (EDO). Sin embargo, los brotes epidémicos producidos por cualquier causa y con implicaciones comunitarias sí se deben notificar al Sistema de Vigilancia Epidemiológica Andaluz (SVEA).

El 17 de febrero de 2003 se comunicó por el Hospital Universitario Virgen de las Nieves de Granada un caso de neumonía atípica en un varón de 21 años ingresado por fiebre con antecedente de exposición a pájaros exóticos y el fallecimiento de un hermano por sepsis. Al tener ambos hermanos una tienda de animales de compañía (pajarería) se sospechó un brote de psitacosis y se procedió a su investigación.

Los objetivos de este trabajo son describir el origen del brote y sus características.

\section{SUJETOS Y MÉTODOS}

Estudio descriptivo del brote de psitacosis ocurrido en Granada durante los meses de febrero-marzo de 2003. Para detectar nuevos casos se alertó a los servicios de urgencias y centros de salud de la posibilidad de que personas en contacto con aves exóticas acudieran con síntomas respiratorios y/o fiebre.

Se definió como expuestas a las personas próximas a las aves adquiridas en cualquiera de las pajarerías que habían recibido pájaros del Centro de importación y cuarentena, proveedor de la tienda de ambos hermanos, así como a otras personas que en aquellos momentos poseían pájaros exóticos o disponían de establecimientos de venta de pájaros y que habían comprado en dicha empresa.

Los casos se clasificaron según los criterios del $\mathrm{CDC}^{6}$ :

- Caso confirmado: presenta clínica compatible con psitacosis y está confirmado por laboratorio (serología, detección molecular en muestras de tejidos).

- Caso probable: la enfermedad clínica es compatible con psitacosis, existe exposición a pájaros pero sin haberse realizado serología.

- Caso descartado: presenta clínica compatible con psitacosis y pruebas de laboratorio negativas.

A las personas sintomáticas se les realizó historia clínica, análisis de sangre y serología/detección molecular del microorganismo; además se les pautó tratamiento específico.

En el análisis estadístico se realizó un análisis de frecuencias absolutas y relativas de las variables descriptivas.

Las muestras clínicas humanas se analizaron en los hospitales de referencia. El estudio de las muestras de tejido pulmonar fue- 
ron analizados en el Instituto Andaluz de Patología y Microbiología de Málaga. Las muestras animales se analizaron en el laboratorio del Ministerio de Agricultura en Algete (Madrid).

\section{RESULTADOS}

De las 17 personas identificadas como expuestas (incluyendo al sujeto que había fallecido) el $53 \%$ eran varones y el $47 \%$ mujeres, con una edad media de 30 años. Nueve personas refirieron síntomas $(53 \%)$ y el resto permanecieron asintomáticas $(50 \%$ varones y $50 \%$ mujeres).

Entre los 9 sujetos expuestos y sintomáticos, el $100 \%$ refirieron fiebre; tuvo tos un $44,4 \%$; cuadro gripal el $44,4 \%$; un $22,2 \%$ amigdalitis; $22,2 \%$ náuseas; neumonía, sepsis, insuficiencia respiratoria (en un paciente con EPOC), petequias, exantema, y diarrea un $11,1 \%$ respectivamente.

El 88,8\% de los casos recibió tratamiento antibiótico con tetraciclinas/macrólidos.

Se realizó serología para Chlamydiophila psitacci a 11 personas, $68,75 \%$ de los expuestos, resultando positiva en dos de ellos $(18,18 \%)$. Entre las personas con síntomas se confirmó serológicamente el diagnóstico en el $28,5 \%$ de los casos. Los resultados de la serología entre los pacientes sintomáticos y asintomáticos se refleja en la tabla 1.

Las muestras de tejido pulmonar del paciente fallecido fueron enviadas al Institu- to Andaluz de Patología y Microbiología en Málaga por el Instituto Nacional de Toxicología (Ministerio de Justicia) de Sevilla, donde se realizó la detección molecular del microorganismo, dando resultado positivo al detectarse presencia de DNA procedente de Chlamydiophila psitacci.

La confirmación diagnostica de psitacosis entre los pacientes sintomáticos estudiados fue del 37,5\% (3 de 8), siendo negativo el resultado de la serología en la totalidad de las personas expuestas asintomáticas. Siete de los sujetos expuestos trabajaban en la pajarería donde se inició el brote. En éstos, con excepción de los dos casos iniciales, las serologías fueron negativas (4), 3 de ellos asintomáticos y 1 con sintomatología, al cual se le indicó tratamiento con macrólidos.

La inspección realizada por los veterinarios de la Delegación de Agricultura permitió detectar el origen del brote en un Centro de Importación y Cuarentena con importación reciente de aves, en el que se identificó la ruptura de la cuarentena preceptiva. Las actuaciones y medidas de control se resumen en la tabla 2.

La Delegación de Agricultura impuso cuarentena a 22 núcleos zoológicos relacionados con la compra de pájaros en la empresa mayorista (Centro de Importación y Cuarentena) y en aquellos en los que no justificaban su origen.

De las 70 muestras tomadas por la Delegación de Agricultura 60 procedían de las cloacas de los animales y 10 eran aves ente-

Tabla 1

Serología de las personas que habían tenido contacto con aves exóticas

\begin{tabular}{|l|c|c|c|}
\hline & Síntomáticas & Asintomáticas & Total \\
\hline Serología negativa & 5 & 4 & 9 \\
\hline Serología positiva & 2 & 0 & 2 \\
\hline No se realizó & 1 & 4 & 5 \\
\hline Total & 8 & 8 & 16 \\
\hline
\end{tabular}


Tabla 2

Medidas realizadas durante el brote de Psitacosis

\begin{tabular}{|c|c|c|}
\hline Dirigidas a & Realizadas por & Actuaciones \\
\hline Personal sanitario & $\begin{array}{c}\text { Delegación de Salud } \\
\text { Servicio de Medicina Preventiva } \\
\text { del Hospital Virgen de las Nieves }\end{array}$ & $\begin{array}{l}\text { - Información a los Servicios de Urgencias } \\
\text { (Hospital, Atención primaria) } \\
\text { - Comunicación como alerta al SVEA } \\
\text { - Definición de caso } \\
\text { - Realización ante paciente sintomático y } \\
\text { exposición a pájaros:radiografía de tórax, } \\
\text { serología y tratamiento } \\
\text { - Si asintomático, no iniciar tratamiento } \\
\text { preventivo. }\end{array}$ \\
\hline Población general & $\begin{array}{c}\text { Delegación de Salud } \\
\text { Delegación de Agricultura }\end{array}$ & $\begin{array}{l}\text { - Recomendaciones higiénicas } \\
\text { - Comunicación a la prensa } \\
\text { - Información a la asociación de vecinos } \\
\text { sobre la enfermedad y mecanismos de } \\
\text { transmisión }\end{array}$ \\
\hline Exposición laboral & $\begin{array}{c}\text { Delegación de Agricultura } \\
\text { Ayuntamiento } \\
\text { Hospital (Servicios de Medicina } \\
\text { Preventiva, Urgencias, } \\
\text { Neumología) }\end{array}$ & $\begin{array}{l}\text { - Inspección a la pajarerías } \\
\text { — Toma de muestras } \\
\text { — Información por parte de la forense } \\
\text { - Recomendaciones higiénicas a los } \\
\text { propietarios } \\
\text { - Encuesta epidemiológica y tratamiento a } \\
\text { todo caso sintomático. }\end{array}$ \\
\hline Animales & $\begin{array}{c}\text { Consejería } \\
\text { Delegación de Agricultura } \\
\text { Ayuntamiento }\end{array}$ & $\begin{array}{l}\text { - La Consejería realiza las actuaciones } \\
\text { pertinentes en las pajarerías de otras } \\
\text { provincias } \\
\text { - Se recomienda el sacrificio de los } \\
\text { animales o aislamiento y tratamiento. }\end{array}$ \\
\hline $\begin{array}{l}\text { Centro de } \\
\text { importación }\end{array}$ & $\begin{array}{l}\text { Servicio de Protección de la } \\
\text { Naturaleza de la Guardia Civil } \\
\text { (SERPRONA) } \\
\text { Juzgados }\end{array}$ & $\begin{array}{l}\text { - Ante el incumplimiento de la cuarentena } \\
\text { impuesta en su momento, la guardia civil } \\
\text { realiza las actuaciones pertinentes. } \\
\text { - Medidas judiciales }\end{array}$ \\
\hline
\end{tabular}

ras; todas fueron analizadas en el laboratorio de referencia del Ministerio de Agricultura en Algete (Madrid). El resultado fue positivo (detección cualitativa directa de antígeno de Chlamydiophila) en 7 de las 60 muestras de cloaca $(11,67 \%), 10 \%$ del total de muestras. En las 10 muestras de aves enviadas la determinación de anticuerpos fue positivo a títulos bajos en 7, siendo la detección de ácidos nucleicos (técnica PCR) positiva en una de ellas.

\section{DISCUSIÓN}

Se trata de un brote de psitacosis de carácter laboral relacionado con un foco común de contagio (exposición a aves enfermas), es decir, aquellas pajarerías que habían adquirido pájaros del Centro de Importación de aves exóticas implicado en el brote.

Los tres casos confirmados trabajaban con pájaros. Ninguna de las personas de las 
que se tuvo conocimiento que tenían animales domésticos se confirmó como afectada de psitacosis, tanto las que presentaron algún tipo de síntoma como las asintomáticas.

Entre los sujetos expuestos con algún tipo de síntomas, la clínica más frecuente ha sido un cuadro respiratorio semejante a clínica gripal (fiebre, tos, rinorrea, odinofagia), salvo los dos casos confirmados e iniciales que se presentaron en forma de sepsis y neumonía. La frecuencia de la afectación fue similar entre varones y mujeres.

El brote ocurrió en invierno coincidiendo con la epidemia de gripe, por lo que la sintomatología referida por la mayoría de los sujetos expuestos podía corresponder a este proceso, quedando finalmente confirmados 3 casos y el resto descartados. De igual forma este mismo hecho puede haber llevado a que otros casos de sintomatología leve hayan podido pasar inadvertidos.

El estudio ha puesto en evidencia el incumplimiento de la cuarentena y tratamiento que en su momento se impuso a las aves importadas por sospecha de psitacosis, durante la inspección rutinaria que los veterinarios de la Delegación de Agricultura realizaron con anterioridad en dichos locales.

El mecanismo de transmisión de la psitacosis es por inhalación, a través de la aerosolización de las secreciones infectantes, polvo de las plumas o excretas desecadas. El periodo de incubación de la enfermedad es de 514 días y la gravedad se extiende desde inaparente a enfermedad sistémica acompañada de neumonía, e incluso puede llegar a ser mortal. Por tanto, ante una neumonía inexplicada y/o que no responda a betalactámicos es importante pensar en ella y buscar una historia de contacto con pájaros. La transmisión persona-persona es muy rara, pero se ha descrito como posible en algún caso de psitacosis familiar en la que no se ha podido demostrar el antecedente de contacto con pájaros ${ }^{7}$. También se han descrito casos de psitacosis tras contacto esporádico, como el de visitas a parques públicos ${ }^{8}$, agentes de aduanas $^{9}$, en el que siete agentes belgas desarrollaron neumonía atípica, siendo confirmada la enfermedad serológicamente en dos de ellos. El riesgo de contraer psitacosis era 2,8 veces superior en los que habían estado en contacto con los periquitos durante más de dos horas que en aquéllos que estuvieron menos tiempo. También se han documentado casos en mataderos de aves ${ }^{10}$. Chlamydiophila psitacci también se ha aislado en patos, ovejas, pollos e incluso en gatos ${ }^{11}$.

En Argentina ${ }^{12}$, en la provincia del Chaco, se comunicó un brote de psitacosis en julio del año 2002, con 18 casos con clínica y epidemiología compatible con psitacosis y confirmados por detección de $\operatorname{IgM}$ para Chlamydiophila. Los enfermos tenían relación con la captura de aves silvestres que habían sido posteriormente regaladas a varias familias.

En fechas próximas ocurrió un brote familiar de psitacosis con 5 afectados en Cádiz ${ }^{13}$, sin relación aparente, ya que los pájaros fueron comprados en una pajarería de esta provincia que no se encuentra en la relación de establecimientos que compraron en el Centro de Importación causante del brote.

En Granada se notificó un brote anterior de psitacosis de ámbito familiar en 1999, (López Hernández B. Técnico de Salud de Epidemiología del Distrito Sanitario Granada. Informe de brote de Psitacosis. Granada 1999) el cual afectó a tres miembros de una familia con clínica de neumonía que habían adquirido unas cotorras 9 días antes. Ante la sospecha de que éstas hubiesen sido el vehículo de transmisión se realizó su examen por la Delegación provincial de Agricultura y el Ayuntamiento de Granada, y la inspección del establecimiento para detectar posibles irregularidades en la adquisición de las aves exóticas. Las cotorras fueron retiradas y analizadas sin lograrse ningún aislamiento. También se realizó difusión de la noticia en 
la prensa local y recomendaciones a los propietarios de mascotas para la prevención y educación sanitaria. Con tratamiento adecuado los afectados evolucionaron favorablemente.

En el brote descrito, la noticia del fallecimiento del primer caso afectado apareció en la prensa local y se aprovechó esta circunstancia para difundir recomendaciones a la población general.

En 1990 en Vall de Uxó (Castellón) hubo un brote de neumonía atípica, probablemente psitacosis, en 4 personas relacionadas familiarmente y que días antes habían adquirido una cotorra en un mercado ambulante. La serología no confirmó claramente esta hipótesis al no haberse encontrado seroconversión en ninguno de los sujetos, aunque la clínica, epidemiología y respuesta al tratamiento orientaron a este diagnóstico. En la prueba serológica realizada al animal se encontró un título bajo, lo que sugería el contacto con Chlamydiophilas pero no confirmaba infección persistente ni excreción ${ }^{14}$.

Muchos países han notificado y divulgado brotes de psitacosis familiares y laborales. En todos ellos se resalta la importancia de la buena coordinación y colaboración con los veterinarios, para identificar la fuente de infección y proceder al control del foco, de hecho se han descrito recaídas de la infección al no eliminar la causa ${ }^{5,6,14}$.

Tras el estudio del brote se obtuvieron las siguientes conclusiones: fue un brote de psitacosis de carácter profesional con implicación de la empresa distribuidora por incumplimiento de cuarentena. Se recomienda incluir la psitacosis en el diagnóstico diferencial de toda neumonía atípica o toda neumonía que no responde al tratamiento inicial con betalactámicos.

Se resalta que la buena coordinación y colaboración con la Delegación de Agricultura permitió el control del riesgo, y posiblemente la aparición de casos nuevos. La información y educación de la población (tanto de los trabajadores relacionados con las aves como de la población general) es necesaria para evitar futuros casos y para lograr un diagnóstico precoz en caso de enfermedad.

\section{BIBLIOGRAFÍA}

1. Jones RB, Batteiger BE. Introducción a las enfermedades por clamidias. En: Enfermedades Infecciosas. Principios y Práctica. Mandell, Bennett, Dolin editores. Madrid: Editorial Panamericana; 1997.p. 2412-5.

2. Cuevas Martínez R. Pájaros argentinos. PsitacosisOrnitosis. 2000. Disponible en: (citado noviembre 2001).

3. Organización Panamericana de la salud. Psitacosis. En: Chin J. El control de las enfermedades transmisibles. Washington DC: Organización Panamericana de la salud; 2001.p. 524- 7.

4. Pérez-Trallero E, Montes M, Cilla G. Importancia de Micoplasma, Coxiella, Chlamydia y Legionella en la neumonía grave de la comunidad. Med Clin (Barc) 1998; 110 (Supl 1): 71-6.

5. Bañó Aracil M, González Morán F, Bertomeu Blanch F, Bellido Blasco J, Sánchez Linares E, Arnedo Pena A, et al. Brote familiar de neumonía por psitacosis. Med Clin (Barc) 1992; 99: 262-4.

6. CDC. Compendium of measures to control Chlamydia psittaci infection among humans (psittacosis) and pet birds (avian chlamydiosis), 2000. MMWR 2000; 49:1-17.

7. Ito I, Ishida T, Mishima M, Osawa M, Arita M, Hashimoto T, Kishimoto T. Familial cases of psittacosis: possible person-to-person transmission. Intern Med 2002; 41 (7): 580-3.

8. Schlossberg D. Chlamydia psittaci (Psitacosis). En: Mandell, Douglas, Bennett. Enfermedades infecciosas. Principios y práctica. Madrid: Editorial médica Panamericana;1997; 2435-7.

9. Schrijver K. Un brote de Psitacosis en agentes de aduana belgas. Eurosurveillance report 1995; 1-3. Disponible en: www.eurosurveillance.org/em.

10. Center for Diseases Control. Epidemiologic notes and reports psittacosis at a turkey processing plantnorth Carolina, 1989. MMRW 1990; 39 (27). 460- 
461, 467-469. Disponible en: www.cdc.gov/ mmwr/preview/

11. Peeling R W, Brunham R C. Chlamydiae as pathogens: new species and new issues. Emerg Infect Dis 1996; 2 (4): 1-13.

12. Ministerio de la Salud de la Nación República Argentina. Dirección de Epidemiología. Boletín Epidemiológico Periódico 2002; 1(4). Disponible en: (21-02-2003).
13. Delegación Provincial de Salud de Cádiz. Sección de Epidemiología. Sistema de Vigilancia Epidemiológica de Andalucía (SVEA). Informe semanal viernes 7 de febrero de 2003 .

14. Instituto de Salud Carlos III. Centro Nacional de Epidemiología. Estudio de un brote de neumonía atípica probablemente de Psitacosis. Boletín Epidemiológico Semanal. 1990; 1.869: 225. 
